\title{
TRES ANEXOS DE LA CRISIS \\ SOBRE EL MUNDO DE LA VIDA
}

\section{Nota de introducción}

por Javier SAN MARTÍN

La razón de ofrecer la traducción de los presentes anexos de La crisis de las ciencias europeas es dar a la publicidad el trabajo realizado para una de las sesiones del Seminario de la Sociedad Española de Fenomenología sobre el mundo de la vida. Al no haber incluido dichos anexos en la edición que de Las crisis se ha hecho en la Editorial Crítica, nos pareció oportuno hacerlos públicos. Los textos XVII y XIX proceden de las pP. 144-147 y 178 del número 1 del manuscrito husserliano $\mathrm{K}$ III, que abarca los textos que Husserl escribió para La crisis. El número XVIII es una hoja suelta que Husserl añadió al manuscrito de la tercera parte de La crisis. La época exacta de los textos ha podido ser establecida con precisión por el editor de Hua. XXIX, de manera que los Anexos XVII y XIX proceden ambos de mayo de 1936. En ese momento está Husserl trabajando intensamente en el manuscrito de La crisis, cuya primera parte (parte I y II del actual texto) la envió a Praga en enero de ese año. Justo en ese mes de enero tuvo que soportar dos graves humillaciones, la retirada de la capacidad docente, en base a las leyes de Nuremberg, y la orden del Ministerio de darse de baja en la Sociedad de Filosofía de Belgrado. Sin embargo, parece que Husserl ya ha encontrado su camino. Todas las angustias de los tres años anteriores no han conseguido quebrarlo y ahora ya tiene como meta definitiva de su vida terminar la reelaboración de las conferencias de Praga de noviembre de 1935 como su última gran aportación, o como le dice a Félix Kaufmann, como su «testamento político». A 
finales de febrero pensaba Husserl tener ya lista la segunda entrega, que sería la parte III del actual texto. A mitades de marzo su hijo y su yerno ya han salido de Alemania, para terminar en los Estados Unidos. El cambio de horizonte no puede menos de tranquilizar a Husserl, por más que la preocupación no deje nunca de estar presente. El 14 de marzo Malvina Husserl escribe a Albrecht que Husserl está embarcado en un gran viaje; «de las conferencias [de Praga] va a salir un gran escrito que contiene el centro del trabajo de su vida. Sólo tengo miedo de que se abrume» ${ }^{1}$. Efectivamente, sin que sepamos las fechas con precisión, Husserl tuvo a milades de marzo una pleuresía, que le apartó de su trabajo mis de un mes, y después le obligó a hacer un mes de convalecencia en Rapallo; pues bien, los manuscritos de que aquí tratamos proceden o bien del mismo Rapallo o de unos días inmediatamente posteriores, y están pensados para el $\$ 34$ de La crisis.

El primer texto trata de explorar la diferencia entre el mundo particular, por ejemplo, de una profesión, que tiene su verdad y su ontología, y el mundo de la vida común pero atemático mientras estamos en el primero, así como el tema aporético que surge del hecho de que el mundo que la ciencia prevé en su trabajo es un mundo particular que se sostiene en el mundo más amplio de la vida. El segundo texto es una exposición sobre uno de los puntos fundamentales del mundo de la vida, el darnos los criterios de realidad, es en la experiencia del mundo ordinario, del mundo de la doxa, donde aprendemos a conocer algo como existente. El tercero plantea el problema de la contraposición del mundo científico y el de la vida, sobre todo cuando se lleva esa contraposición al seno mismo de la filosofia.

Quiero agradecer expresamente a los Archivos de Husserl en Lovaina asi como a la Editorial Kluwer la desinteresada autorización para la traducción y publicación de estos textos, así como el interés mostrado en la cuidadosa lectura que de la traducción han hecho con valiosas sugerencias para mejorarla.

${ }^{1}$ Briefwechsel, IX, p. 125. 


\section{ANEXO XVII}

\section{(A los $\iint 33$ ss)}

Siempre vivimos en el mundo de la vida de un modo consciente. Normalmente no hay ningún motivo para convertir en explícitamente temático el mundo de un modo universal. Conscientes del mundo como un horizonte, vivimos para nuestros fines particulares, bien sean momentáneos y cambiantes o como una meta que nos guía permanentemente. En este caso puede tratarse de una meta que hemos elegido nosotros mismos como una profesión vital, para ser la dominante en nuestra vida activa, o puede ser tal que en cierta manera hayamos sido introducidos en ella por la educación. En este caso se constituye un horizonte cerrado del "mundo". En tanto que seres humanos con una profesión podemos permitirnos ser indiferentes a cualquier otra cosa, teniendo ojos sólo para este horizonte como nuestro mundo y para sus propias realidades y posibilidades, -aquellas que existen en este mundo- es decir, tenemos puesta la mirada sólo en lo que es "realidad" aquí (lo que es correcto, verdadero en relación a esta meta) o "irrealidad" (lo incorrecto, lo erróneo, lo falso).

Que toda esta vida eficaz y todo este mundo de trabajo se mantiene dentro del mundo siempre existente de un modo obvio, en el sentido más universal y pleno de mundo de la vida; que la actividad particular y las obras presuponen su "verdad y falsedad" en términos de lo que existe y no existe, de lo que es correcto y erróneo en la esfera más amplia del ser -esto permanece fuera de nuestro interés, aunque en la vida de los intereses particulares hacemos uso de lo que existe en ina esfera más amplia, de acuerdo a nuestras necesidades particulares. Así cuando vivimos temáticamente sólo en un mundo particular (bajo la reglamentación de la meta superior que lo "realiza"), el mundo de la vida es atemático para nosotros; y en tanto permanece tal, tenemos nuestro mundo particular, el único mundo que es temático en cuanto tal, como nuestro horizonte de interés. 
Puede ser aquí que esta meta directriz sea en última instancia una meta común, es decir, una tarea vital personal que es una tarea parcial (si es que se puede hablar de "parte" en este caso) dentro de una tarea común, de modo que el individuo personal que emprende un trabajo funcione en concurso y conscientemente, así para cada uno de los "participantes" en la empresa común.

La vida dirigida por metas, que es la vida profesional de los científicos, cae claramente bajo la caracterización general que se acaba de hacer, junto con el "mundo" que es suscitado [erweckte] en la comunalización de los científicos (a través del encadenamiento de las sucesivas generaciones de investigadores) como el horizonte de los trabajos científicos. Pero los trabajos científicos tienen una característica típica que no pertenece necesariamente a todos estos mundos y sus fines. Todos los trabajos pertenecientes al mundo científico con su específico y común sentido de ser (ser real y verdadero $y$, por otra parte, incorrecto, falso para todas las personas de la comunidad), no sólo constituyen una multiplicidad y una copertenencia ontológica; más bien los trabajos particulares, los resultados científicos particulares llegan a ser premisas, sillares para trabajos de un nivel más alto $\mathrm{y}$ esto por necesidad y in infinitum y a la vez de tal modo que los trabajos de la ciencia [se juntan] en un trabajo total coherente, el sistema teórico (la teoría contenida en el libro de texto ideal). El mundo científico, el horizonte ontológico de los científicos, tiene el carácter de una obra única o edificio que crece in infinitum, que las generaciones de científicos, que le pertenecen correlativamente, están construyendo sin término. El sistema teórico aquí, sin embargo, es una obra de verdad predicativa, en la que está suscitado correlativamente, como un sustrato universal, el ser-verdadero teórico en ese "dominio" -bajo la más alta idea-fin, la verdaderamente determinante, de lograr un ser incondicionalmente verdadero (una idea situada en el infinito). En el sistema teórico de afirmaciones este "ser en la verdad" es el sobre qué [Worüber] idéntico subyacente en el sentido teórico de las mismas como el concepto ideal del ámbito y de sus seres. 
El mundo científico - la teoría sistemática- y lo que está contenido en él como existiendo en verdad científica (en ciencia natural, en la teoría universal, su naturaleza, naturaleza que vale como sustrato de las proposiciones, de las proposiciones formales), como todos los otros mundos teleológicos, pertenece él mismo al mundo de la vida, exactamente como todos los seres humanos y todas las comunidades humanas en general, y sus fines humanos, ambos individuales y comunales, con todas sus correspondientes formaciones de la actividad [Werkgebilde, working structures] pertenecen también a él. Pero esto vale también para la filosofía en el viejo sentido, cuyo "universo" teórico es el mundo en el más amplio sentido. Tenemos especiales motivos para mencionarla en último lugar. Cada uno de estos "mundos" tiene su universalidad particular determinada por el objetivo profesional; cada uno tiene el horizonte infinito de una cierta "totalidad". Pero todas esas totalidades se insertan en el mundo que rodea todo lo que existe y todas las totalidades existentes a la vez que todos sus fines y todos los seres humanos teleológicos y las civilizaciones. Todo esto tiene su lugar en él y todo lo presupone. ¿Qué significa esto? ¿Qué significa para el mundo de la "filosofia"? ¿No surge aquí una necesaria y a la vez peligrosa duplicidad en el significado del "mundo", del ámbito de la filosofía, que, después de todo, supone que tiene como su tema el mundo pleno y total, junto con todos los mundos particulares antes mencionados? Y la teoría universal buscada por la filosofía debe ser, después de todo, también la teoría de la existencia de la humanidad y de sus fines y obras, e incluso debería abarcarse a sí misma, porque ella misma es una formación teleológica del ser humano.

No nos dejemos confundir. Hay que distinguir el ámbito que precede a todos los fines y a la idea-fin que guía de un modo unitario, en el más alto nivel, aquel ámbito en términos de lo que pretende este fin, en términos del dominio del que trata, de lo que tiene de antemano a la vista, para actuar en relación con él de un modo teleológico y crear formaciones de la actividad; y por otro lado el ámbito de los fines, el ámbito de lo que ya ha sido obtenido teleológica. mente y de lo que está todavía por alcanzar en su propia universalidad, 
el horizonte de realidades concebido antes o después como suscitado y para ser suscitado en particular por las ciencias. La naturaleza predonada -el ámbito del mundo de la vida- la naturaleza corpórea, es aquel que es familiar al hombre corriente en la vida cotidiana y que se puede conocer "con más detalle" pero que él simplemente no tiene ningún motivo para destacar y considerar de una manera coherente en su carácter de unidad abstracta, como la ciencia natural se propone hacer. Para él ella es la esfera predonada de ser para la que desea realizar algo nuevo: teoría para la naturaleza, ser teóricamente verdadero, determinación predicativa -bajo la idea de incondicionalidad, verdad universalmente válida. Este es el ámbito del científico natural orientado puramente hacia los fines de su profesión; y dentro de este ámbito a su vez hay que distinguir: lo que ha sido ya establecido teóricamente y en un cierto sentido el horizonte total de la ciencia al que eso pertenece, pero en la situación en la que eso ha sido establecido por ella; por otra parte, lo que se debe establecer como nuevas tareas para la ciencia, puesto que lo ya terminado es siempre a la vez fundamento sobre el que construir teóricamente después, a saber, para hacer nuevas preguntas y responderlas.

Finalmente, en el caso de la "filosofía" (en el viejo sentido), debemos distinguir igualmente el mundo simplemente, el que siempre es autoevidente, el conocido-desconocido mundo de la vida, como el ámbito universal, para el cual se pone una meta universal -teoría, ciencia para este mundo-; y la vida del científico que es apropiada para poner este fin, su horizonte de intereses y sus horizontes de "realidades", de resultados, no sólo aquellos bajo el título de "naturaleza existente en verdad científica", sino también aquel del "mundo en general". Esta verdad es una estructura teleológica teórica relacionada al mundo que existe en la vida precientífica de un modo incuestionado, en certeza de ser y realidad autoevidente; pero no es este mundo mismo.

Está claro lo que constituye aquí la diferencia radical. El mundo de la vida es el mundo que está constantemente predonado, constantemente válido y existente de antemano, pero no válido por algún proyecto de investigación, de acuerdo con alguna meta universal. 
Todas las metas lo presuponen, incluso de antemano lo presupone la meta universal del conocimiento en el sentido científico; y en el curso del trabajo científico siempre lo presupone de nuevo como un mundo existente en su propio modo, pero de todos modos existiendo. El mundo científico (naturaleza en el sentido de ciencia natural, mundo en el sentido de filosofía como ciencia positiva universal) es una estructura teleológica extendida hacia el infinito - una estructura de los hombres presupuestos, para el mundo de la vida presupuesto. Si ahora hay que hacer evidente que el mundo de la vida mismo es una "formación" [Gebilde], no es, sin embargo, una "formación teleológica", aunque a su ser, que precede a toda meta, pertenezcan los seres humanos, como nosotros los encontramos y llegamos a conocerlos sin más, con todas sus intenciones y sus obras, que, en tanto hechas por el ser humano, en adelante por supuesto también pertenecen al mundo de la vida.

Aquí se produce de nuevo una confusión: todo el mundo práctico, toda ciencia, presupone el mundo de la vida; como formación teleológica se opone al mundo de la vida que siempre fue y sigue siendo "por sí mismo". Por otra parte todo lo que la humanidad ha hecho y está haciendo (individual o comunitariamente) es una parte del mundo de la vida: así se suprime la oposición. Pero esto sólo puede llevar a confusión, porque los científicos, como todos aquellos que viven comunitariamente bajo una meta profesional ("meta vital") no tienen ojos más que para sus propios fines y horizonte de trabajo. Por más que el mundo de la vida sea el mundo en que ellos viven, incluso al que pertenecen todas sus "obras teóricas", y por más que usen los elementos del mundo de la vida, que, como lo tratado, es precisamente el "fundamento" del tratamiento teórico, el mundo de la vida mismo no es precisamente el tema, ni como lo predonado a ellos en cada caso, ni como el que posteriormente recibe su trabajo; y así no es su tema, en una sinopsis completa, el universo de lo que es, aquello que siempre está para nosotros en un movimiento incesante de relatividad y es suelo para todos los proyectos particulares, fines, horizontes de fines $y$ horizontes de trabajo para metas de un nivel más alto. 
En tanto el científico habla como científico está en la actitud científica, pensando dentro del horizonte de su meta teórica, por así decir, pensando dentro de él, y a la vez teniéndolo como horizonte en validez universal privilegiada, como el horizonte actual de su interés profesional. El resto del mundo, la totalidad del mundo que eo ipso incorpora todas las formaciones teleológicas humanas dentro de sí mismo como totalidad del mundo, queda fuera de sus intereses. El ser universal pleno del mundo de la vida -especialmente en su [función de hacer posible] su mundo teórico [del científico] y la correspondiente predonación que le pertenece- esta completamente desconsiderado.

Pero ahora la pregunta paradójica: ¿no se puede convertir el mundo de la vida, el mundo del cual somos conscientes en la vida como el mundo de todos nosotros, sin hacerlo tema universal de investigación, estando siempre entregados a nuestras metas e intereses profesionales diarios, momentáneos, ya sean individuales o universales, no se puede, en un cambio de actitud, tener una visión universal de él y no se puede tratar de conocer qué y cómo es en su propia movilidad y relatividad, hacerlo tema de una ciencia universal, pero tal que de ninguna manera tenga la meta de una teoría universal en el sentido en que ésta era buscada por la filosofía histórica y las ciencias? 


\section{ANEXO XVIII}

\section{$($ al $\$ 34)$}

Puede ser que la posición de tareas [Aufgabenstellung] de la ciencia objetiva haya entrado en el horizonte de la humanidad filosófica no por un azar histórico, sino por necesidad, como sicndo la primera en poder ser comprendida. Pero puede que lo que es primero para nosotros no sea la ciencia primera en sí, que como tal sólo en la repercusión unilateral de los siglos y también en la repercusión sobre el conjunto del devenir histórico de la humanidad podía motivar reflexiones en las que podía aparecer el sentido nuevo de la ciencia, ante todo su sentido como ciencia del mundo de la vida en tanto que suelo para la ciencia objetiva, en cierta medida, por tanto, como medio de ayuda (como remedio). Eso es lo que espero mostrar, así como en el desarrollo consecuente, que no nos podemos quedar en el descubrimiento del mundo de la vida como tema teórico (a saber, el mundo predonado a la ciencia objetiva como autoevidente) y que esta ciencia de un género nuevo, la ciencia del mundo de la vida, engloba, en definitiva, de un modo inevitable en la universalidad de tareas que constituyen su cientificidad propia, todos los problemas provistos de sentido en cuanto al ser y al conocimiento de la verdad. No sólo todas las cuestiones del conocimiento que juegan su papel en la vida (en la vida precientífica y extracientífica), sino también, en un nivel superior, todas las preguntas de la ciencia objetiva. Pero entonces en una modalidad de fundación que conduce a nuevas dimensiones, y por ellas mismas, en la medida en que se abren en su profundidad infinita, a una ciencia universal que encierra en ella todos los conocimientos imaginables y todos los problemas del conocimiento - todos los problemas de la razón.

La idea de la verdad objetiva y del conocimiento objetivo está determinada de antemano por su contraste con la idea de la verdad y del conocimiento propias de la vida extracientífica, que, en sus validaciones ontológicas y en el horizonte abierto universal que constantemente comporta de un modo dado a la conciencia [bewußt- 
seinsmäßig], designa el concepto de mundo de la vida con el que chocamos en primer término. En la vida, por tanto en este mundo, vale como "efectivamente" existente lo que se atestigua por la experiencia. Toda mención, toda validación en el sentido más amplio, si no es ya una mención de experiencia [crfahrende Meinung] (por ejemplo, una percepción simple), debe ser verificada por la experiencia. Para la vida extracientífica en la experiencia lo mentado está dado como él mismo ahí, en la percepción como presente él mismo, en el recuerdo como representificado él mismo, y así para todos los modos de la intuición, de la experiencia en el sentido más amplio, aquello que uno tiene delante de los ojos como eso mismo. La experiencia verifica, atestigua la mención justamente por el hecho de presentar lo objetivo mismo, invocándolo a dar testimonio. Pero también la experiencia necesita verificación en la medida en que también ella, de un modo confiado, comporta momentos de co-mención que aún no son momentos de experiencia efectiva. La experiencia se verifica por la continuación de la experiencia, por la continuación de la experiencia activa, y porque en esa continuación lo que está co-mentado y pre-mentado continúa verificándose en el modo de la experiencia.

A la experiencia en tanto que co-mención, pertenece también la inducción, que, por encima de lo mentado como él mismo en una experiencia unitaria, señala hacia otras objetividades y sus posibles experiencias. No hay diferencia esencial en uno y otro caso; por ejemplo, en la experiencia de una cosa, los lados co-mentados de esta misma cosa, que van más allá de los lados percibidos cada vez, eso es en el fondo una inducción, por decirlo así, una inducción interior frente a la siempre concomitante referencia externa a la co-presencia [Miteinander] de otras cosas en tanto que experienciables, en su inductividad interna. La experiencia efectiva es la que en definitiva verifica el ser mentado y la verificación misma da al ser que antes sólo era mentado el carácter de ser efectivo y verdadero, confirmación de "es así". En este "es así", en este ser en verdad con que el que hace la experiencia se satisface en su situación, se ventila el conocimiento, su conservación y desde ahora la posibilidad de disponer siempre de nuevo de ese ser como efectivamente existente. Pero la 
vida es un continuo movimiento y eso implica ante todo que aparecen discordancias, que la verificación sufre una ruptura y que ahora se tiene experiencia de algo pero no encajando, ya no como algo que cumple la pre-mención, sino que se le opone. Entonces, en lugar de la certeza del ser y de la confirmación, se produce más bien duda, mera pretensión, y eventualmente en definitiva la experiencia negativa, la de la apariencia negadora [nichtigen], de la tachadura de] ser. Pero tales experiencias negativas en lo particular no suprimen el acuerdo normal universal, que siempre se restablece de nuevo. El amplio conjunto de lo que concuerda absorbe por así decirlo la nueva experiencia, gracias a las correspondientes modificaciones de opiniones, y de antemano se está seguro de que toda duda puede ser superada, y que a todo ser corresponde, o le puede ser producido, otro ser apropiado, a todo ser negativo, un ser real apropiado. $Y$ también, lo que de antemano juega un papel continuo, en el acuerdo comunicativo a partir del desacuerdo, por tanto como posibilidad de corregirse mutuamente y de llegar a una verdad en la comunidad. El mundo, en tanto que el horizonte común con valor de ser y de las cosas existentes, tiene siempre y de antemano el valor ontológico de un poder ser dado en experiencia de un modo concordante y de poder ser corregido mediante intercambios recíprocos en todas direcciones hasta el acuerdo. Esta descripción, por lo demás muy basta, muestra que el mundo de la vida está en perpetuo movimiento, tal que está en un movimiento de continua relatividad en la validación y referencia a quienes viven juntos, que tienen juntos el mundo, como soportes y ejecutores de la validación, como quienes tienen menciones, o quienes tienen experiencias unos con otros y unos frente a otros, pero siempre corrigiendo de nuevo a los otros y pudiendo corregirles.

Este mundo de la vida no es otro que el de la simple $\delta o ́ \xi \alpha$, tradicionalmente tratada de un modo tan despectivo. En la vida extracientífica no es en absoluto desvalorizada de semejante manera; ahí significa una esfera de validaciones seguras; de aquellas que dan posibilidad y sentido a la vida total de intereses [gesamten Interessenleben] del ser humano en todos sus objetivos. A todos los objetivos, 
por tanto también a los teóricos de las ciencias objetivas, pues éstas comportan efectivamente "evidencias", de las que el científico hace continuo uso. Hablando de un modo universal, el mundo de estas cosas entendidas de un modo autoevidente como existentes y como pudiendo ser demostradas verdaderas y efectivas en el modo de la $\delta o ́ \xi \alpha$, este mundo es el suelo sólo sobre el cual se puede desarrollar toda ciencia objetiva. En una palabra, el mundo de la vida, este mundo "puramente" subjetivo y relativo es, en su flujo incesante de validaciones de ser, con sus cambios y correcciones, es, por inuy paradójico que pueda parecer, el suelo sobre el que la ciencia objetiva edifica sus formaciones de verdades "definitivamente válidas", "eternas", esas formaciones de los juicios absolutamente válidos una vez por todas y para todo el mundo.

Ciertamente ella sabe que eso es un simple ideal y tal vez confiese que la meta se encuentra en el infinito. Pero tiene esa idea directriz, que pertenece a su propiedad esencial, y por mucho que sus teorías estén en un movimiento histórico de perfeccionamiento, como en una relatividad propia de su validez, se trata de juicios sobre el suelo del mundo de la vida, es decir, sobre el fundamento de una validación universal de ser que se produce en la movilidad de la vida de validación de los sujetos, con la correspondiente certeza, que opera verificaciones, a través de la experiencia cotidiana y de la confirmación de la experiencia. 


\section{ANEXO XIX}

\section{(al $\$ 34$ e)}

La oposición: mundo científico, mundo del científico, que no es sino el universo teleológico [zweckvolles] de la verdad científica, que sólo tiene el ser científicamente verdadero; y mundo de la vida, en el que esas metas y obras, como todos los demás, confluyen. Pero los científicos mismos son seres humanos en el mundo de la vida, seres humanos entre los demás. El mundo de la vida es mundo para todos, y por eso las ciencias, que son en primer lugar los mundos de los científicos, existen para todos los seres humanos como nuestro producto, nuestra adquisición (enunciados, teorías), existen para todos, así como el mundo de la vida es para todos, de una manera subjetivo-relativa. La filosofía debe ser la ciencia universal del ser, debe englobar a todo ser en su verdad; esto significaba tradicionalmente crear para el conjunto del mundo de la vida, este mundo subjetivo-relativo, una teoría objetiva, suponerle la idea teleológica [Zweckidee] universal de una verdad definitivamente válida y poderla realizar en tanto que idea teleológica. ¿Es esto en general una empresa dotada de sentido? ¿Se puede decidir esto sin interrogarse por el ser propio del mundo de la vida? ¿Está incluido en tal filosofía el ser del mundo de la vida? ¿no hay una tensión entre el mundo de la vida y el mundo científico, entendidos ambos como existentes? No sólo por el hecho de que los científicos son por sí mismos seres humanos en el mundo de la vida, perteneciendo a una humanidad que está en el inundo de la vida; sino también porque siendo ellos mismos seres humanos en el mundo de la vida, no siempre están interesados en la ciencia y ocupados por ella, sino que tienen muchos otros intereses que presuponen como existente el mundo de la vida con sus diversos modos de predonación.

El mundo de la vida es subjetivo-relativo; no es un universo ontológico obtenido por la idea teleológica universal "mundo existenten $^{n}$, como si no existiera antes de que se desarrollara esta idea y como si esta pretendida idea teleológica no supusiera el mundo de la vida. Pero este es algo subjetivo y algo que vale ontológicamente en la relatividad para los hombres en tanto que "sujetos para el mundo", $y$ estos sujetos siguen siempre viviendo en sus intereses, intereses instintivos, pero también de representación y de voluntad -intereses 
teleológicos. Siempre siguen teniendo metas y siempre termina lo que se persigue confluyendo en el mundo de la vida como lo suscitado [Erwecktes]; mundo predonado de la vida que siempre se ha hecho [ist geworden] a partir de los intereses humanos, siempre algo así como nuestro mundo de conciencia.

Convertir a los seres humanos y a la humanidad universal en tema ino es eo ipso transformar en tema toda esta vida de conciencia con todo el contenido subjetivo? Pero itiene entonces un sentido tratarla como una temática teleológica objetiva y querer así llegar al mundo relativo-subjetivo que siempre está presupuesto (que es una efectuación de conciencia de los seres humanos)?

*t

El sentido y la posibilidad de una ciencia objetiva universal -su posibilidad, no su posibilitación práctica, su posibilidad como praxis dotada de sentido, que persigue un objetivo dotado de sentido, que comporta consigo métodos dotados de sentido- se convierte en un problema, y a la vez también se convierte en problema una filosofía, una teoría efectivamente universal del ser, que abarque a todo ente de tal modo que ninguno se le escapara de las manos como presupuesto, es decir, como nunca cuestionado en su ser propio, en su confirmación propia de ser.

¿Son una y otra idea de la filosofía idénticas o no son más bien diferentes por principio; la una contradictoria, la otra, dotada de sentido y necesaria? Y con ello ¿no se hace la pregunta de si para la existencia de la humanidad en tanto que humanidad, que en cuanto tal es de un modo práctico, es decir que sólo puede vivir [ihr Leben könnend] en una praxis que tenga éxito, no se da la necesidad esencial de crearse en la filosofía el órgano de la autorreflexión última y entonces < conocer> en esa única filosofía posible, el modo cómo puede vivir, cómo puede, en su horizonte de infinidad y de infinidad de destinos, conseguir la "felicidad" [glückselig werden]? ¿Y no pertenece esto al reino de la ciencia filosófica?

Traducción de Javier San Martín y de Jesús Miguel Díaz Álvarez 